\section{ACLARACIÓN EDITORIAL SOBRE UN CASO DE PUBLICACIÓN REDUNDANTE}

\section{EDITORIAL CLARIFICATION ABOUT A CASE OF REDUNDANT PUBLICATION}

\section{J. Jhonnel Alarco ${ }^{1, a}$, Franco Romaní1,b}

Sres. Lectores. La Revista Peruana de Medicina Experimental y Salud Pública (RPMESP) tiene establecida una política editorial frente a faltas éticas como la publicación redundante o duplicada ${ }^{(1)}$. Como en la mayoría de las revistas científicas nuestras políticas editoriales prohíben la publicación redundante; sin embargo, pueden darse excepciones. El Comité Editorial de la RPMESP ha tomado las decisiones y recomendaciones descritas en esta publicación basándose en las recomendaciones del Comité Internacional de Editores de Revistas Biomédicas (ICMJE) y del Comité de Ética en Publicación (COPE).

Nuestro Comité Editorial puede aceptar alguna publicación redundante, si esta es justificable y obedece a algunas razones. Una de ellas es que por la significancia de los hallazgos el manuscrito deba ser republicado en un idioma o país distinto, o en una revista de otra especialidad o profesión, en ambos casos el objetivo es alcanzar a una audiencia diferente, la cual no tendría acceso a los hallazgos, sino fuera por la republicación. En ambos casos, los editores deben estar informados y de acuerdo con la publicación en las revistas involucradas ${ }^{(2)}$.

Altamirano-Gamarra et al. (3) informan a la RPMESP sobre un nuevo caso de publicación duplicada o redundante, con una detallada descripción y explicación de los criterios que definen esta práctica. Ante esta comunicación, nuestro Comité Editorial inició el procedimiento establecido para estos casos ${ }^{(4)}$. Comprobamos el grado de redundancia, así como la modificación de la composición de autores, variación del título, cambios en las secciones del manuscrito, modificaciones en la bibliografía, y no referencia de la publicación primaria ${ }^{(1)}$.

Procedimos a solicitar una explicación al autor de la publicación aludida. La carta al editor que contiene la réplica de Waters et al. (5) (autor corresponsal) confirmaría los criterios para definir este caso como una publicación

\footnotetext{
1 Revista Peruana de Medicina Experimental y Salud Pública, Instituto Nacional de Salud. Lima, Perú.

a Editor adjunto; ${ }^{\mathrm{b}}$ editor científico.

Recibido: 04/09/2018 Aprobado: 05/09/2018 En línea: 20/09/2018
}

Citar como: Alarco JJ, Romaní F. Aclaración editorial sobre un caso de publicación redundante. Rev Peru Med Exp Salud Publica. 2018;35(3): 5456. doi: 10.17843/rpmesp.2018.353.3905 redundante (Tabla 1) ${ }^{(6)}$, y consideramos, que los autores no han brindado una explicación satisfactoria que demuestre lo contrario.

Es responsabilidad del autor poner en conocimiento a los editores de ambas revistas, y justificar la necesidad de la publicación redundante, así como adjuntar copias de los manuscritos o publicaciones previas ${ }^{(1,2)}$. En el presente caso, el manuscrito ingresó al proceso editorial de la RPMESP incluyendo una declaración jurada que indicaba que el manuscrito no había sido enviado a otra revista. El Comité Editorial consideró que sea sometido a revisión por pares, las observaciones de los revisores fueron absueltas por los autores, y finalmente fue aceptado para publicación, en un proceso editorial que duró aproximadamente cinco meses.

Nuestro proceso editorial no identificó la publicación redundante, a pesar de que el artículo de Public Health Nutrition (13 de septiembre de 2016) estuvo disponible en diferentes bases de datos electrónicas (Web of Science, PubMed $\AA$, Scopus $\AA$, Embase $\AA$, entre otras) durante cinco meses, periodo en el que se dio nuestra revisión por pares, culminando con su aprobación el 22 de febrero del 2017 y publicado un mes después. Es probable que no se haya detectado la primera publicación por encontrarse en inglés y con un título diferente.

Los editores de las revistas científicas podrán actuar con equidad con los autores, si es que cuentan con políticas editoriales sobre publicación redundante, que ayuden a simplificar las decisiones en este tema, y que aseguren un abordaje consistente para todos los casos ${ }^{(2)}$. Nuestras instrucciones para autores son actualizadas regularmente (https://goo.gl/GQjeZC), y establecen que «las propuestas de publicación no deben haber sido publicadas previamente de manera parcial o total, ni enviado simultáneamente a otras revistas científicas, en cualquier idioma». En las instrucciones también instamos a los autores a seguir las recomendaciones del COPE. Sin embargo, en vista de los recientes casos detectados de publicación redundante, el Comité Editorial de la RPMESP actualizará en su página

Tabla 1. Criterios para definir publicación redundante*

\begin{tabular}{ll}
\hline Criterios $^{*}$ & Definición \\
\hline 1 & Los tamaños de muestra son similares \\
2 & La metodología es idéntica o casi idéntica \\
3 & $\begin{array}{l}\text { Los resultados son similares } \\
\text { Al menos un autor es común en ambos } \\
\text { estudios }\end{array}$ \\
5 & $\begin{array}{l}\text { Poca o ninguna información relevante es } \\
\text { aportada }\end{array}$ \\
\hline
\end{tabular}

* Para la definición de publicación redundante se deben de cumplir todos los criterios

Fuente: Modificado de Kumar et al. (6) 
web, políticas editoriales específicas para este tipo de mala práctica en publicación (incluyendo las situaciones especiales en que la publicación redundante puede ser aceptada, y el procedimiento para solicitarlo).

La presente aclaración consensuada del Comité Editorial de la RPMESP constituye una expresión de preocupación, y una declaración de publicación redundante, realizada en estricto cumplimiento de las recomendaciones del COPE. Nuestra intención es informar a otros investigadores y autores sobre este caso, proteger la credibilidad de nuestras políticas editoriales, y potencialmente frustrar posibles beneficios que podrían tener los autores por la publicación redundante ${ }^{(2)}$. Agradecemos a nuestros lectores por informarnos activamente y los alentamos a seguir notificando las malas prácticas en publicación científica. Consideramos que es responsabilidad de todos (autores, editores, lectores e instituciones de investigación) fomentar la integridad científica e instamos a los autores a seguir estrictamente las pautas establecidas para una conducta científica responsable.

Contribuciones de autoría: JJA y FR participaron en la concepción y redacción. Ambos autores participaron en la revisión final y aprobación del manuscrito.

Fuentes de financiamiento: autofinanciado.

Conflictos de interés: Los autores declaran ser miembros del Comité Editorial de la Revista Peruana de Medicina Experimental y Salud Pública.

\section{REFERENCIAS BIBLIOGRÁFICAS}

1. Mayta-Tristán P, Curioso WH. Política editorial ante la detección de una publicación redundante. Rev Peru Med Exp Salud Publica. 2009;26(1):5-8.

2. Scientific Style and Format: The CSE Manual for Authors, Editors, and Publishers, Council of Science Editors. Eighth Edition [Internet]. Chicago: The University of Chicago Press; 2018. p. 16-17. [citado el 5 de septiembre de 2018]. Disponible en: https://press. uchicago.edu/ucp/books/book/chicago/S/bo13231737.html

3. Altamirano-Gamarra J, Aguirre-Alcántara P, Silva-Barboza C, Reyes-Rodríguez N, Merma-Flores G. Nuevo caso de publicación duplicada o redundante en idiomas diferentes. Rev Peru Med Exp Salud Publica. 2018;35(3):542-3.

4. Comité de Ética en Publicación (COPE). Flujograma: Qué hacer si sospecha que una publicación es redundante o duplicada (b) Sospecha de publicación redundante en un artículo publicado [Internet]. Hampshire: COPE; 2018. [citado el 5 de septiembre de 2018]. Disponible en: https://publicationethics.org/files/Spanish\%20 \%281\%29.pdf

5. Freire WB, Waters WF, Rivas-Mariño G. Réplica: nuevo caso de publicación duplicada o redundante en idiomas diferentes. Rev Peru Med Exp Salud Publica. 2018;35(3):544.

6. Kumar AS, Beyersdorf F, Lazar HL, Patterson GA, Weisel RD. Joint statement on redundant (duplicate) publication by the Editors of the undersigned cardio-thoracic journals. Asian Cardiovasc Thorac Ann. 2015;23(7):773.

Correspondencia: Franco Romani

Dirección: Cápac Yupanqui 1400, Jesús María. Lima 11, Perú.

Teléfono: (+51) 990630678

Correoelectrónico:fromani@ins.gob.pe

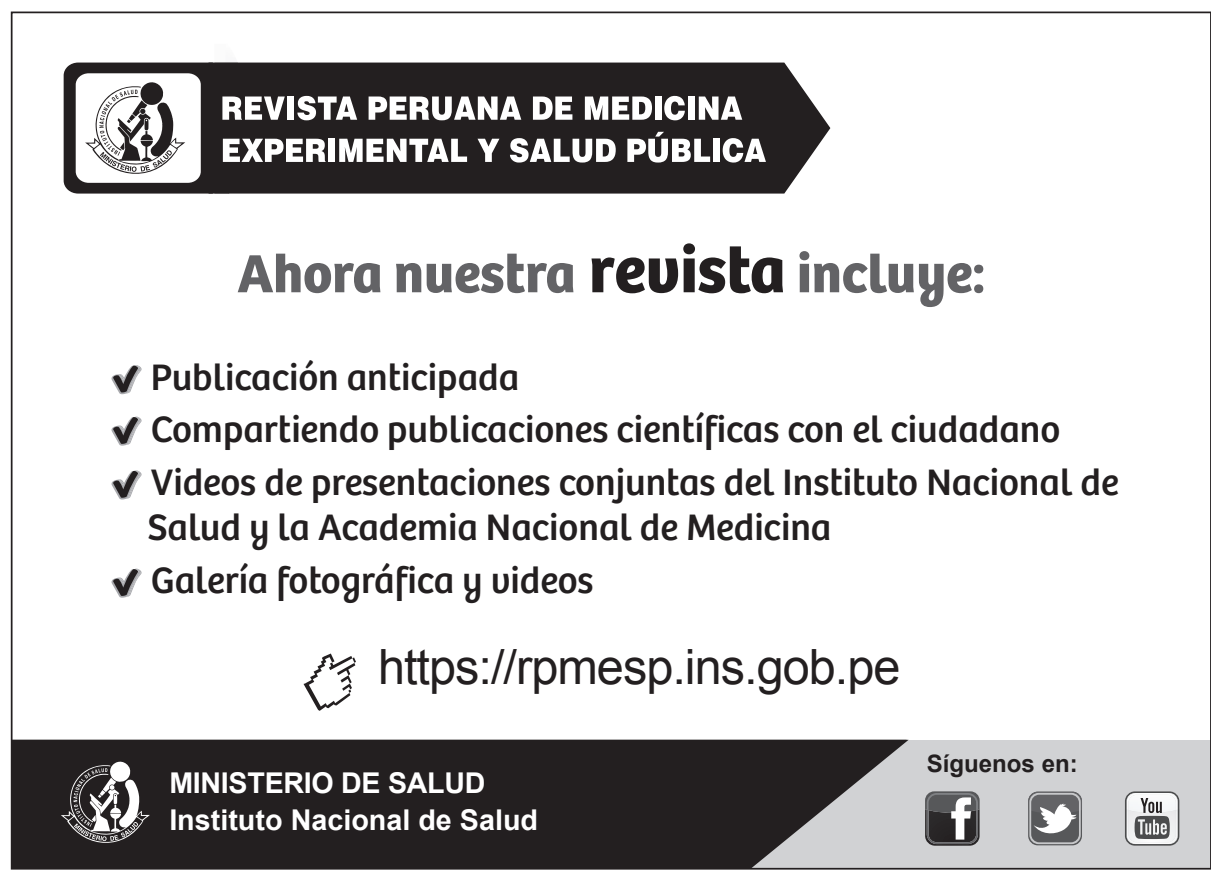

